\title{
Los servicios audiovisuales por Internet de las universidades españolas: Estudio de las dimensiones unidireccionales e interactivas
}

\author{
José Borja ArJona MARTín \\ Centro Universitario Villanueva (adscrito a la U. Complutense de Madrid) \\ jbarjona@villanueva.edu \\ María GALMÉS CEREZO \\ ESIC Business and Marketing Madrid \\ galmescerezo.maria@gmail.com
}

\begin{abstract}
Resumen
Los servicios audiovisuales para redes IP se encuentran ya plenamente consolidados. La universidad no es ajena ante las oportunidades que podría ofrecer un canal audiovisual propio por Internet. Es por ello que pretendemos analizar los servicios audiovisuales para la Web, de un número significativo de universidades españolas, en busca del nivel de implantación de funcionalidades interactivas y unidireccionales. Con este objetivo general, se propone un modo de análisis cuantitativo que ofrezca valores comparables en cada uno de los casos de estudio.
\end{abstract}

Palabras Clave: IPTV, Web, Universidad, Unidireccionalidad, Interactividad

\section{Internet Television and Video for Spanish Universities: A study of unidirec- tional and interactive dimensions}

\begin{abstract}
Audiovisual services for IP networks are now fully consolidated. The opportunities that could provide an Internet audiovisual channel are very interesting for universities. We analyze audiovisual web services from a significant number of spanish universities, in search of the interactive and unidirectional level of their capabilities. With this general aim it is proposed a quantitative analysis model that provides comparable values in each of the study cases.
\end{abstract}

Key Words: IPTV; Web, University, Unidirectionality, Interactive

Referencia normalizada:

Arjona Martín, J. B.; Galmés Cerezo, M. (2013) Los servicios audiovisuales por Internet de las universidades españolas: Estudio de las dimensiones unidireccionales e interactivas. Historia y Comunicación Social. Vol. $18 \mathrm{~N}^{\circ}$ Especial Octubre. Págs. 471-480.

Sumario: 1. Introducción. 2. Objetivos. 3. Metodología. 4. Resultados. 5. Conclusiones. 6. Referencias Bibliográficas 


\section{Introducción}

La red de Internet se ha consolidado como un medio más para la distribución de servicios audiovisuales. Para la universidad española, este medio ha supuesto una oportunidad para la creación de un espacio de información, divulgación y de apoyo a la docencia.

Las redes IP proporcionan múltiples posibilidades para la distribución de vídeo: IPTV, vídeo bajo demanda, televisión conectada, redes sociales de vídeo, etc. Muchos son los términos empleados y sus definiciones. Sin embargo, el trabajo de Simpson y Greenfield (2009) - uno de los más destacados en este campo - proporciona una exhaustiva ordenación del ámbito de la televisión y el vídeo por IP, el cual representa el marco de referencia que utilizaremos para la acotación de nuestro objeto de estudio.

Dichos autores clasifican las diferentes modalidades de distribución de vídeo IP en cuatro categorías: IPTV, IPVOD, Internet TV e Internet Video.

Inicialmente los términos IPTV e Internet Video parecían suficientes para definir la panorámica de los servicios audiovisuales por redes IP. Asimismo las diferencias entre ambos términos parecían claras y suficientes. Sin embargo, en un contexto de vertiginosos cambios tecnológicos, las fronteras entre ambos términos se difuminan y surgen nuevos conceptos para hacer referencia a los usos y contenidos más recientes.

Desde el año 2006 han nacido gran variedad de nuevos servicios que necesitan de nuevas categorías y términos que los definan. Para completar el ámbito de los servicios audiovisuales a través del protocolo de Internet, Greenfield y Simpson, proponen la creación de dos nuevas categorías situadas entre IPTV e Internet Video. A estas categorías las han denominado Internet TV e IPVOD.

La expresión IPTV se utiliza para designar los servicios proveídos a través de redes privadas y que ofrecen paquetes (bajo suscripción) de canales de emisión continua. A estos servicios se accede mediante Set-Top Box (STB) conectado al televisor, o sin la "caja propietario" en el caso de televisores con conectividad.

Por su parte, IPVOD (IP Video On Demand) ofrece una combinación de entrega para el televisor (mediante STBs) y el ordenador personal. Proporciona producción profesional de vídeo a la carta mediante algún sistema previo de pago (suscripción o pago por visión).

Los términos Internet TV e Internet Vídeo designan los servicios audiovisuales cuyo principal dispositivos de acceso es un ordenador personal o aparato portátil (smartphones o tablets). Su distribución se realiza por medio del protocolo http (Web), haciendo uso de redes de distribución públicas, generalmente Internet.

Internet TV comprende una amplia oferta de canales de emisión continua, generalmente de producción profesional, si bien cada vez es más común encontrar contenido amateur. Como ejemplo, ambos autores citan la iniciativa NASA TV (www.nasa. gov/multimedia/nasatv/schedule.html), la cual proporciona horas de programación 
en directo y diferido cada día, incluyendo retransmisiones de lanzamientos y aterrizajes de sus misiones espaciales. El usuario tan sólo conecta con el canal, no puede elegir entre la programación. CNN.com Live (www.cnn.com/live) y Bloomberg Live TV (www.bloomberg.com) ofrecen un servicio similar, en los que el usuario conecta con el stream (flujo de vídeo) en tiempo real.

Las iniciativas de Internet Vídeo, por el contrario, constituyen amplios archivos de clips de vídeo (de duración variable) producidos especialmente por los usuarios, contenido conocido como User-Generated Content (UGC). Muchos son los casos conocidos que podrían ser definidos como Internet Video: por ejemplo, el paradigmático caso de YouTube; pero también Dailymotion, Vimeo, BlipTV, e incluso Facebook según Simpson y Greenfield.

En base a estos criterios, el término "portal" se adapta a los nuevos servicios de televisión y vídeo creados para su distribución por la Web. Nuevos medios en los que podemos ver los productos televisivos de siempre y otros generados exclusivamente para la red, con nuevas posibilidades complementarias.

Expuesto lo anterior, el presente estudio se centra en aquellos medios que cumplan con los criterios definidos bajo los términos Internet TV e Internet Vídeo, impulsados desde las principales universidades españolas, tanto públicas, como privadas: servicios audiovisuales distribuidos por Internet para toda la comunidad universitaria, por medio de un navegador (uso del protocolo http) y con acceso por ordenador personal o dispositivos móviles. El usuario puede acceder a canales de programación continua y contenidos a la carta, de producción profesional o amateur.

Una vez explicados los criterios con los que acotaremos nuestro objeto de estudio, cabe detenernos en el segundo concepto a analizar: la interactividad aplicada a servicios audiovisuales.

En base a la aproximación teórica adoptada en el estudio de la interactividad (Kim y Sawhney, 2002), podríamos entenderla como un entorno de intercambio de información, entre figuras que alternan sus roles en una estructura reticular. $\mathrm{O}$ bien, podría concebirse desde una perspectiva enfocada en las cualidades tecnológicas del medio, el cual marcará el alcance de la interactividad proporcionada.

La televisión constituye el tradicional ámbito de estudio de la interactividad aplicada a servicios audiovisuales, y su gran dificultad reside en que no existe aún un marco general que describa una clasificación, generalizada y comúnmente aceptada, de los modelos de interactividad en televisión. Microsoft utiliza la siguiente clasificación de los modelos de televisión interactiva (Gawlinski, 2003):

- Televisión mejorada (Enhanced Television): Servicios que habilitan opciones de participación a través de los contenidos audiovisuales.

- Televisión con Internet: Permite conectarse a Internet por medio del televisor.

- Televisión personalizada: Los espectadores pueden seleccionar entre un catálogo de contenidos o bien grabar con dispositivos DVR. 
- Televisión conectada: Sistemas de interconexión de dispositivos con los que poder compartir una misma información.

La organización de investigación sobre consumo, The Henley Centre, propone su clasificación de televisión interactiva:

- Distribución interactiva: En la que el usuario tiene el dominio sobre la reproducción del contenido.

- Información interactiva: El espectador puede acceder a varios tipos de información por medio del televisor.

- Participación interactiva: Durante la emisión de los programas o espacios publicitarios se proporcionan diferentes opciones para el espectador. Por ejemplo, jugar en un programa o elegir cámara durante la retransmisión.

En Europa el estándar tradicional utilizado para servicios de televisión digital interactivos es el Multimedia Home Platform (MHP), con el que extender el uso de nuevas aplicaciones a la televisión en abierto, compitiendo en cierto sentido con el PC conectado a Internet (competencia desigual, en muchos sentidos). No obstante, desde el año 2009, un consorcio europeo de empresas del sector audiovisual (European Broadcasting Union) y compañías tecnológicas, trabajan por generalizar el estándar HbbTV (Hybrid Broadcast Broadband Television). Una solución tecnológica que nos ofrece un televisor con conexión directa a Internet (como canal de retorno), sin intermediario (descodificador o Set-Top Box), que mantiene, además, el canal de envío de información tradicional (TDT, satélite). HbbTV no es ningún concepto nuevo, sólo una propuesta estándar para generalizar lo que las empresas fabricantes ya están comercializando bajo el concepto de SmartTV.

Sin embargo, hasta la fecha, la Web, como canal audiovisual, aprovecha en gran medida las capacidades de participación que ofrece Internet, además de erigirse como un campo de innovación de formatos y contenidos audiovisuales que, en muchos casos, trascienden de la red a la televisión generalista o temática.

\section{Objetivos de investigación}

El objetivo general de este estudio consiste en identificar tanto funcionalidades unidireccionales, como interactivas, en los medios audiovisuales por Internet de las universidades españolas, con el fin de comparar ambas dimensiones en cada uno de los casos de estudio.

Entendemos por funcionalidades unidireccionales (o dimensión unidireccional) aquellas acciones que transcurren en un solo sentido, de medio a usuario. Por el contrario, las funcionalidades interactivas permiten a los usuarios interactuar con el medio y con otros usuarios.

En base a lo expuesto, plateamos los siguientes objetivos específicos: 
- Definición de los indicadores de función.

- Clasificar los indicadores de función en base a su naturaleza unidireccional o interactiva.

- Según lo anterior definiremos las dimensiones "unidireccionalidad" e "interactividad".

- Para cada caso de estudio comparar sus niveles en cada una de las dimensiones.

\section{Metodología}

Con el objeto de determinar cuál es el nivel de interactividad y unidireccionalidad que ofrecen los diferentes casos de estudio, hemos definido dos dimensiones ("unidireccionalidad" e "interactividad"), como explicábamos previamente, en base a las cuales clasificaremos los indicadores de función según su naturaleza unidireccional o interactiva.

Hemos procedido a la suma de la inclusión de dichos indicadores en cada caso de estudio, de tal modo que la adición de las frecuencias nos ofrecerá un valor cuantitativo con el fin de analizar las diferencias entre las distintas dimensiones. Dichas dimensiones no son excluyentes, es decir, cada caso de estudio obtiene puntuación tanto en "unidireccionalidad" como en "interactividad", porque pueden presentar funciones recogidas en ambas dimensiones. Tener un mayor peso en una u otra dimensión nos ayudará a definir el nivel de interactividad en cada caso. Por tanto, estas dos dimensiones no pueden ser tomadas como polos o extremos de un continuo, sino como características independientes que podrán tener una mayor o menor presencia.

Para la recogida y análisis de los datos hemos utilizado el programa SPSS Statistics V20, utilizando un sistema binario -asignación de valores 0 y 1- para indicar si cada caso de estudio incluye o no cada una de las funcionalidades definidas. Asignamos valores nominales a cada una de las funcionalidades de manera que otorguen un significado cuantitativo a los objetos, con el fin de utilizar estos valores en cálculos estadísticos.

Finalmente, con el fin de contrastar las dimensiones "unidireccionalidad" e "interactividad" fue necesario realizar un cálculo ponderado de los valores medios en ambas dimensiones, puesto que sus escalas de medida son diferentes.

Las universidades españolas que poseen servicios de vídeo y televisión para redes IP, según los criterios establecidos por Simpson y Greenfield, son:

- CEU Media Televisión (ceumedia.es)

- Universidad de Deusto (deusto.tv) 
- Universidad de Valladolid (uva.es/opencms/contenidos/serviciosAdministrativos/gabinetesApoyoTecnico/servicioMediosAudiovisuales/Mediateca)

- Universidad Europea Miguel de Cervantes (uemc.es/es/uemctv)

- Universidad de Alicante (aplicacionesua.cpd.ua.es/album)

- Universidad Autónoma de Barcelona (uab.es/videos)

- Universidad Camilo José Cela (mediacampus.ucjc.edu)

- Universidad Castilla la Mancha (uclmtv.uclm.es)

- Universidad de Coruña (udctv.es)

- Universidad de Málaga (infouma.uma.es)

- Universidad de Sevilla (tv.us.es)

- Universidad de Valencia (mediauni.uv.es)

- Universidad Internacional de La Rioja (tv.unir.net)

- Universidad Internacional Menéndez Pelayo (uimp20.es/tv)

- Universidad Jaume I (svideo.uji.es)

- Universidad Nacional de Educación a Distancia (canaluned.com)

- Universidad de Oviedo (mediateca.uniovi.es/inicio)

- Universidad del País Vasco (ehutb.ehu.es)

- Universidad Rey Juan Carlos (chaplin.urjc.es/urjctv)

- Universidad Politécnica de Valencia (upv.es/pls/oreg/rtv_web.Ppal?p_idio$\mathrm{ma}=\mathrm{c}$ )

- Universidad de Vigo (tv.uvigo.es)

\section{Resultados}

Siguiendo los objetivos planteados en este artículo, y una vez revisados los casos de estudio, pasamos a describir las funcionalidades más extendidas en el conjunto de los servicios objeto de análisis:

1. Emisión continua: Selección, coordinación y ordenación previa, y ajena al usuario, de programas con una duración determinada.

2. Descarga de vídeo: La descarga de contenido en diversos formatos como Windows Media, Quicktime, Realplayer, o bien para distintos dispositivos como IPod, PSP o teléfonos móviles.

3. Canales: Programas independientes que elaboran y gestionan su propio contenido, en vivo y/o diferido, bajo el marco general de un servicio concreto.

4. Relación de contenidos: El usuario hallará, junto al reproductor, una lista de vídeos relacionados con el tema principal del documento original. 
5. Vídeo en directo: Hacemos referencia a la transmisión en vivo tanto una señal de televisión digitalizada, como a la transmisión de vídeo desde una webcam.

6. Categorías temáticas: Clasificar sus contenidos de acuerdo con temáticas bien diferenciadas.

7. Carga de vídeo: Ofrece a los usuarios la posibilidad de subir vídeos propios al servicio.

8. Marcas activas o nube de tags: Compone un ranking, presentado a modo de célula, de palabras clave utilizadas por los usuarios como términos de búsqueda.

9. Comentarios: La posibilidad de incluir comentarios en los post (artículos) o vídeos de la plataforma.

10. Recomendación por email: Permite recomendar a otros usuarios contenidos de vídeo a través de correo electrónico.

11. Valoración: Votar sobre la experiencia del usuario con relación a los contenidos o a otros parámetros.

12. Búsqueda de contenidos: Herramientas de búsqueda capaces de localizar documentos afines a los términos usados en los descriptores.

13. Ranking de valoración y votación: A partir de los votos y las valoraciones de los usuarios, ciertas herramientas elaboran listas con los contenidos más votados y más valorados por los internautas.

14. Compartir contenidos en redes sociales y bookmarks: Publicar y realizar referencias de una determinada información (escrita o audiovisual) en servicios de agrupación o redes sociales.

15. Suscripciones RSS: Los formatos RSS permiten redifundir los contenidos mediante los sistemas definidos como agregadores.

16. Vínculos o enlaces relacionados: Redirigir mediante un enlace activo desde una Web a otra relacionada o de interés para la primera.

17. Grupos: Habilitar la posibilidad de crear grupos para la asociación de usuarios bajo una temática común.

18. Chats: Representa las clásicas aplicaciones para la conversación en grupo, en una misma "sala". Reconocemos este indicador como una aplicación especialmente desarrollada para cada caso en concreto, no obstante, la red social Facebook provee, a todo tipo de web que así lo solicite, de una aplicación común llamada Facebook Connect.

19. Foros: Todos aquellos espacios webs dedicados al diálogo y al debate asíncronos en torno a una temática común.

20. Etiquetas (tags): Los vídeos pueden ser etiquetados mediante palabras clave relacionadas con el tema central y secundarios.

21. Insertar (embed): Integrar un vídeo en una Web determinada desde una fuente ajena a la ubicación de destino. 
22. Opciones para el control de la reproducción: Facilitar posibilidades de pausa, avance y retroceso del vídeo.

En el siguiente cuadro se recoge el procedimiento seguido en la adición o suma de funcionalidades que delimitan cada dimensión.

Cuadro 1. Agrupación de funcionalidades según dimensiones

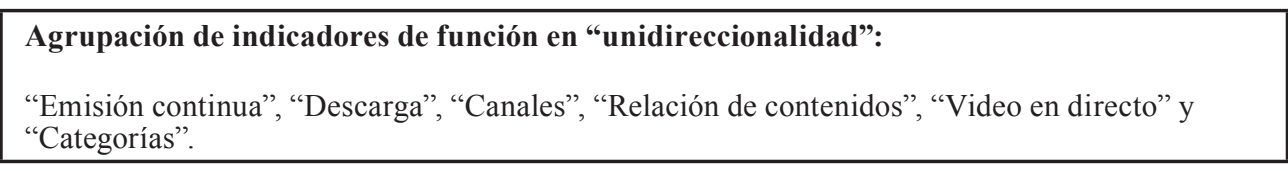

Agrupación de indicadores de función en "interactividad":

"Carga", "Marcas activas o nube de tags", "Comentarios", "Recomendación por e- mail",

"Valoración", "Búsqueda de contenidos", "Rankings valoración y votación", "Compartir contenidos en redes sociales y bookmarks", "Suscripciones RSS", "Vínculos o enlaces relacionados", "Grupos", "Chat", "Foros", "Etiquetas (Tags)", "Insertar (Embed)" y "Opciones para el control de la reproducción".

La tabla 1 muestra los valores medios ponderados en ambas dimensiones para cada uno de los casos analizados. Estas cifras son comparables, de modo que podemos apreciar, en primer lugar, que las funcionalidades definidas como unidireccionales tienen más peso en la mayoría de los casos estudiados.

Siendo el valor máximo 1 (incluye todos los indicadores de función), las cifras máximas en unidireccionalidad corresponden a la Universidad Nacional de Educación a Distancia (UNED), Universidad Internacional Menéndez Pelayo y la Universidad de Vigo, todas ellas con 0,67.

Tabla 1. Medias ponderadas en dimensiones para cada uno de los casos analizados

\begin{tabular}{|l|c|c|}
\hline & Unidireccionalidad & Interactividad \\
\hline CEU Media Televisión & 0,5 & 0,2 \\
\hline Universidad de Deusto & 0,5 & 0,27 \\
\hline Universidad de Valladolid & 0,33 & 0,13 \\
\hline Universidad Europea Miguel de Cervantes & 0,33 & 0,07 \\
\hline Universidad Internacional Menéndez Pelayo & 0,67 & 0,53 \\
\hline Universidad Nacional de Educación a Distancia & 0,67 & 0,27 \\
\hline Universidad de Coruña & 0,33 & 0,13 \\
\hline Universidad Autónoma de Barcelona & 0,17 & 0,2 \\
\hline Universidad Camilo José Cela & 0,33 & 0,4 \\
\hline Universidad Castilla la Mancha & 0,33 & 0,13 \\
\hline Universidad de Alicante & 0,33 & 0,13 \\
\hline Universidad de Málaga & 0,17 & 0,2 \\
\hline
\end{tabular}




\begin{tabular}{|l|c|c|}
\hline Universidad de Sevilla & 0,5 & 0,2 \\
\hline Universidad de Valencia & 0,5 & 0,13 \\
\hline Universidad Internacional de La Rioja & 0,33 & 0,4 \\
\hline Universidad Jaume I & 0,33 & 0,27 \\
\hline Universidad de Oviedo & 0,17 & 0,2 \\
\hline Universidad del País Vasco & 0,5 & 0,2 \\
\hline Universidad Rey Juan Carlos & 0,17 & 0,07 \\
\hline Universidad de Vigo & 0,67 & 0,33 \\
\hline Universidad Politécnica de Valencia & 0,5 & 0,07 \\
\hline
\end{tabular}

Del otro lado, la cifra más alta en interactividad es registrada por la Universidad Internacional Menéndez Pelayo (0,53), seguida por la Universidad Internacional de la Rioja y la Universidad Camilo José Cela, ambas en 0,4. Por su parte, la Universidad Autónoma de Barcelona, Málaga, Oviedo y la Universidad Rey Juan Carlos de Madrid, registran las cifras más bajas en unidireccionalidad.

Las universidades de Málaga, Oviedo, Camilo José Cela e Internacional de La Rioja consiguen mayor media en interactividad, respecto a la dimensión unidireccional, aunque su diferencia no sea muy significativa.

Las 17 universidades restantes obtienen mayor media en unidireccionalidad, siendo en algunos casos una diferencia altamente significativa. Como es el caso de la Universidad Politécnica de Valencia que obtiene 0,5 en unidireccionalidad, frente a un escaso 0,07 en interactividad. También podemos citar los casos de la Universidad de Vigo $(0,67 / 0,33)$, Valencia $(0,5 / 0,13)$, País Vasco $(0,5 / 0,2)$, Sevilla $(0,5 / 0,2)$, UNED $(0,67 / 0,27)$, Europea Miguel de Cervantes $(0,33 / 0,07)$ y San Pablo CEU $(0,5 / 0,2)$.

\section{Conclusiones}

Según los datos alcanzados, las funcionalidades unidireccionales, asociadas a los medios convencionales, dominan la mayor parte de los casos estudiados. Frente a lo que podríamos creer en primer momento, las universidades analizadas aún no aprovechan suficientemente las posibilidades interactivas que ofrece la red.

Probablemente, el concepto reinante tenga más que ver con el uso que hacemos de la televisión convencional, que su combinación con las oportunidades de la Web. Quizá este estudio haya asentado una evidencia empírica acerca de las capacidades de interacción y participación que ofrecen estos servicios, sugiriendo un replanteamiento de sus diseños en pos de la tan deseada interactividad.

Podemos afirmar que las universidades españolas analizadas hacen un uso de la Web, como servicio audiovisual, destinado a proporcionar información y a ofrecer cobertura en diferentes actos (congresos, conferencias, clases magistrales, actos insti- 
tucionales, etc.). Es decir, cubren las funciones elementales de un servicio audiovisual de estas características (institucional). No obstante, en los datos se observa una apertura hacia capacidades más interactivas que, aunque insuficientes, parecen presagiar lo que consideramos como una evolución natural en los medios de distribución de televisión y vídeo por redes IP.

\section{Referencias bibliográficas}

ARJONA MARTÍN, J. B. (2010): Análisis y sistematización de la televisión en Internet (WebTV). Tesis Doctoral. Universidad de Málaga.

CEBRIÁN, C. (2002). Convergencia de la televisión e Internet. Tesis Doctoral. Universidad Complutense de Madrid.

MARTÍNEZ RECIO, A. y CONDE ORTEGA, S. (2012): La televisión (por Internet) de la Universidad de Córdoba, en Revista de Educación Mediática y TIC, $\mathrm{n}^{\circ}$ 1. Disponible en: http://www.edmetic.es/Documentos/Vol1Num1-2012/Angel_ Martinez_y_Sergio_Conde.pdf.

Consultado el 04 de octubre de 2013.

OWEN, B. (1999). The Internet challenge to television. Harvard University Press: Cambridge.

SIMPSON, W. y GREENFIELD, H. (2009): IPTV and Internet Video: Expanding the reach of television broadcasting. Focal Press. Burlington (Massachusetts).

\section{Los autores}

José Borja Arjona Martín. Profesor del área de comunicación en el Centro Universitario Villanueva (adscrito a la Universidad Complutense de Madrid). Profesor en el máster "e-learning y redes sociales" de la Universidad Internacional de La Rioja. Asimismo, pertenece al grupo de investigación "Nuevas Formas Publicitarias y Nueva Economía SEJ-396", de la Universidad de Málaga, en el que ha trabajado para diversas publicaciones científicas.

María Galmés Cerezo. Profesora del área de comunicación de la UNIR (Universidad Internacional de la Rioja). Profesora en el máster "liderazgo y comunicación" de la UMA (Universidad de Málaga). Profesora en el área de marketing en ESIC Business School. Pertenece al grupo de investigación "Nuevas Formas Publicitarias y Nueva Economía SEJ-396" de la Universidad de Málaga. Ha publicado artículos en revistas de comunicación como ADresearch o Pensar la Publicidad, y ha participado como invitada y ponente en varios congresos de Comunicación. 